\title{
Comportamento de Litopenaeus vannamei (Boone) (Crustacea, Decapoda, Penaeidae) em função da oferta do alimento artificial nas fases clara e escura do período de $\mathbf{2 4}$ horas
}

\author{
Cibele S. Pontes \& Maria de F. Arruda
}

Laboratório de Estudo do Comportamento de Camarões, Departamento de Fisiologia, Universidade Federal do Rio Grande do Norte. Caixa Postal 1511, 59072-970 Natal, Rio Grande do Norte, Brasil.E-mail: cspontes@natal.digi.com.br; arruda@cb.ufrn.br

\begin{abstract}
Behavior of Litopenaeus vannamei (Boone) (Crustacea, Decapoda, Penaeidae) in relation to artificial food offer along light and dark phases in a $24 \mathrm{~h}$ period. The few data on the marine shrimp Litopenaeus vannamei (Boone, 1931) behavior related to artificial food offer in feeding trays may result an inadequate feeding by the animal, increasing the cost/benefit relation in shrimp culture and its potential environmental impact. In order to provide tools for optimizing feeding management in shrimp farms, a behavioral study was developed, using $64 \mathrm{~L}$. vannamei juveniles $(7,57 \pm 1,01 \mathrm{~g})$. They were submitted to artificial photoperiods, half of them in reversed cycle, in order to register their behavior during light and dark phases. In established intervals, the following activities were registered before and after food exposition: exploration, swimming, feeding and inactivity (instantaneous sampling). Swimming occurred mostly during the dark phase. Feeding time was higher in the half hour following food offer, especially during light phase. Food offering worked out in both phases as an inductor of substrate exploration, making the shrimp more active in all the intervals following its introduction. KEY WORDS. Applied ethology, shrimp culture.
\end{abstract}

RESUMO. A escassez de dados acerca do comportamento do camarão marinho Litopenaeus vannamei (Boone, 1931) relacionado ao alimento artificial ofertado em comedouros poderá induzir a uma alimentação inadequada, aumentando a relação custo/benefício e os impactos ambientais potenciais do seu cultivo. Objetivando fornecer subsídios para a melhoria do manejo alimentar praticado nas fazendas, foram desenvolvidos estudos comportamentais utilizando 64 juvenis de $L$. vannamei $(7,57 \pm 1,01 \mathrm{~g})$, submetidos a fotoperíodo artificial, metade deles em ciclo invertido, para observação das suas atividades durante as fases clara e escura. A ração foi oferecida em intervalos pré-estabelecidos, registrando-se antes e depois da oferta: exploração, natação, alimentação e inatividade (focal instantâneo). A natação ocorreu preferencialmente durante a fase escura. A alimentação foi mais elevada na primeira meia hora posterior à oferta, com ênfase nos horários da fase clara. $O$ alimento artificial atuou, em ambas as fases, como um indutor da exploração do substrato, tornando os camarões mais ativos em todos os horários posteriores à sua oferta.

PALAVRAS CHAVE. Etologia aplicada, cultivo de camarão.

A carcinicultura marinha brasileira sofreu um grande impulso com a introdução de Litopenaeus vannamei (Boone, 1931) a partir do final da década de 80 . Atualmente, a maioria dos produtores deste camarão utiliza metodologia de cultivo semi-intensivo, caracterizada por densidades populacionais de aproximadamente 30 animais $/ \mathrm{m}^{2}$, pela utilização de aeradores e pela adição de ração aos viveiros em complemento ao alimento natural.

O consumo do alimento artificial é monitorado através da utilização de comedouros (bandejas), o que é considerado, por Martinez-Cordova et al. (1998), um fator de controle da quantidade ministrada, levando à diminuição dos custos com ração e os impactos ambientais causados no solo do viveiro. NunEs et al. (1996) ressaltam que, para um manejo alimentar adequado, deve-se considerar também o tipo de atividade alimentar do camarão, evitando a acumulação de alimento não consumido e a deterioração da água e do solo do viveiro.

No cultivo de camarões marinhos, o gasto com alimento artificial pode representar até $60 \%$ dos custos de produção, em uma tendência que vem se mantendo ao longo do tempo (Sedgwick 1979, Martinez-Cordova et al. 1998, Velasco et al. 1999, SMith et al. 2002, TACON et al. 2002, Cuzon et al. 2004). Uma vez que o manejo alimentar dos cultivos é realizado empiricamente, 
baseado na experiência técnica, é imprescindível o seu aprimoramento. Neste sentido, Huntingford (1991) afirma que a maximização do lucro na exploração comercial de organismos pode ser alcançada através de métodos gerados por pesquisas na área de comportamento animal.

A aplicação de conhecimentos específicos com relação ao comportamento de L. vannamei frente à ração ofertada em comedouros pode constituir uma ferramenta útil na melhoria do seu aproveitamento. Tendo como meta fornecer subsídios para a otimização do manejo alimentar praticado nas fazendas de cultivo, neste estudo avaliamos as atividades comportamentais do camarão marinho L. vannamei com relação à fase (claro/escuro) e horário em que o alimento é ofertado.

\section{MATERIAL E MÉTODOS}

Foram utilizados 64 juvenis da espécie Litopenaeus vannamei, com dois meses $(7,57 \pm 1,01 \mathrm{~g})$, provenientes de viveiro de cultivo, e marcados com anéis de silicone de diferentes cores no pedúnculo ocular para reconhecimento individual. Os experimentos foram desenvolvidos em laboratório, sendo utilizados quatro aquários (50 x $30 \times 40 \mathrm{~cm}$ ) com $30 \mathrm{~L}$ de água do mar cada, em sistema fechado de re-circulação, com aeração constante e filtração contínua através de cascalho e conchas de ostras quebradas. A salinidade da água manteve-se em $34 \%$ e a temperatura em $28 \pm 1^{\circ} \mathrm{C}$.

Para possibilitar a observação simultânea dos comportamentos nas fases clara e escura, os aquários foram submetidos a ciclos artificiais invertidos através de interruptor horário, permanecendo dois deles no claro e dois no escuro (7:00-19:00 h). Cada aquário foi iluminado individualmente através de lâmpadas de 15 watts fluorescente branca para a fase clara e incandescente vermelha para a escura (Rodriguez \& NAYLOR 1972, Hindley 1975), gerando luminosidades médias de 57 e de um lux, respectivamente. Os animais foram colocados na densidade populacional 33 indivíduos $/ \mathrm{m}^{2}$ (quatro por aquário), havendo quatro repetições ao longo do tempo, totalizando oito aquários e 32 indivíduos monitorados para cada uma das fases (claro e escuro). As observações duraram 33 dias para cada uma das repetições e foram iniciadas após 09 dias de adaptação dos animais às condições experimentais.

A alimentação foi realizada com ração peletizada, com 35\% de proteína bruta (Camaronina 35 - Agribrands do Brasil Ltda.), com o equivalente a $10 \%$ da biomassa total presente no aquário. A ração foi ofertada apenas na fase de observação, em comedouros de acrílico transparente $(4 \times 3 \times 2 \mathrm{~cm})$, duas vezes ao dia, uma e nove horas após o início da fase clara ou escura. Foram registradas, através de método focal instantâneo (a cada 60 s), as atividades: a) Natação - deslocamento contínuo horizontal ou vertical, ou ainda suspensão na coluna d'água, através do batimento dos pleópodos; b) Exploração do substrato introdução e retirada contínua dos três primeiros pares de pereópodos (quelados) no substrato, mantendo o cefalotórax inclinado para baixo; c) Alimentação - ingestão do alimento artifi- cial e d) Inatividade - parado com ou sem movimentação dos apêndices locomotores.

Para acompanhamento simultâneo dos quatro aquários foi necessária a participação constante de dois pesquisadores, cada um dos quais contemplando a cada dia, aleatoriamente, um aquário na fase clara e outro na escura. Os indivíduos eram observados durante 15 minutos ( $\mathrm{A}=$ antes da oferta do alimento), e, decorridos 30 minutos, a ração era ofertada. A partir de então, eram monitorados nos 30 minutos posteriores ( = 1) e, após outro intervalo de 30 minutos, voltavam a ser acompanhados por mais 30 minutos $(=2)$, totalizando $480 \mathrm{~h}$ de observação em todo o experimento.

Para efeito de análise, os horários da fase clara foram considerados como manhã (M) e tarde (T) e os da escura como início da noite (IN) e final da noite (FN). Foram avaliadas as freqüências percentuais das atividades ocorridas antes (A), imediatamente após (1) e uma hora após (2) a colocação da ração no comedouro. Uma vez constatada a não aderência dos dados à distribuição normal e a heterogeneidade na variância dos fatores, através dos testes de Shapiro-Wilks e de Levene, respectivamente, utilizou-se o teste de análise de variância não paramétrico de Kruskal-Wallis. Em caso de diferenças significativas aplicou-se o teste U de Mann-Whitney para comparação dois a dois (STEel \& Torrie 1988). Adotou-se o nível de significância de 5\% para avaliação dos resultados, que serão representados graficamente através de sua mediana e amplitude interquartílica (75\% a $25 \%)$.

\section{RESULTADOS}

A freqüência de natação dos animais variou em função dos horários de observação (Kruskal-Wallis, H $(9,45)$ = 368,9; p $=0,00)$, aumentando de imediato a partir do início da fase escura (IN) e mantendo-se elevada em todos os seus horários, que tiveram valores superiores aos da fase clara (U de MannWhitney). A oferta do alimento não interferiu neste padrão de comportamento (Fig. 1).

A exploração do substrato apresentou diferenças com relação aos horários de observação(Kruskal-Wallis, H $(11,45)=$ $440,5 ; \mathrm{p}=0,00)$. Registrou-se o aumento desta atividade em todos os horários posteriores à oferta da ração, nas fases clara e escura, conforme a aplicação do teste U de Mann-Whitney: AM vs $1 \mathrm{M}(\mathrm{U}=461, \mathrm{Z}=-8,43, \mathrm{p}=0,00)$; AT vs $1 \mathrm{~T}(\mathrm{U}=514, \mathrm{Z}=-$ $6,72, \mathrm{p}=0,00)$; AIN vs $1 \mathrm{IN}(\mathrm{U}=540, \mathrm{Z}=-5,57, \mathrm{p}=0,00)$ e AFN vs $1 \mathrm{FN}(\mathrm{U}=433, \mathrm{Z}=-9,51, \mathrm{p}=0,00)$ (Fig. 1).

Houve alterações na freqüência de alimentação de acordo com o horário de observação (Kruskall-Wallis, H $(9,45)=$ $934,73 ; \mathrm{p}=0,00)$. Os primeiros horários da manhã e tarde $(1 \mathrm{M}$ e 1T) mostraram valores semelhantes entre si e superiores a todos os outros. O teste $\mathrm{U}$ de Mann-Whitney detectou ainda percentuais inferiores desta atividade nas segundas meias horas de observação da manhã, tarde, início e final da noite (2M, $2 \mathrm{~T}, 2 \mathrm{IN}$ e $2 \mathrm{FN}$ ), quando comparados aos registrados nas primeiras meias horas (1M, 1T, $1 \mathrm{IN}$ e $1 \mathrm{FN})$ (Fig. 1).

Revista Brasileira de Zoologia 22 (3): 648-652, setembro 2005 

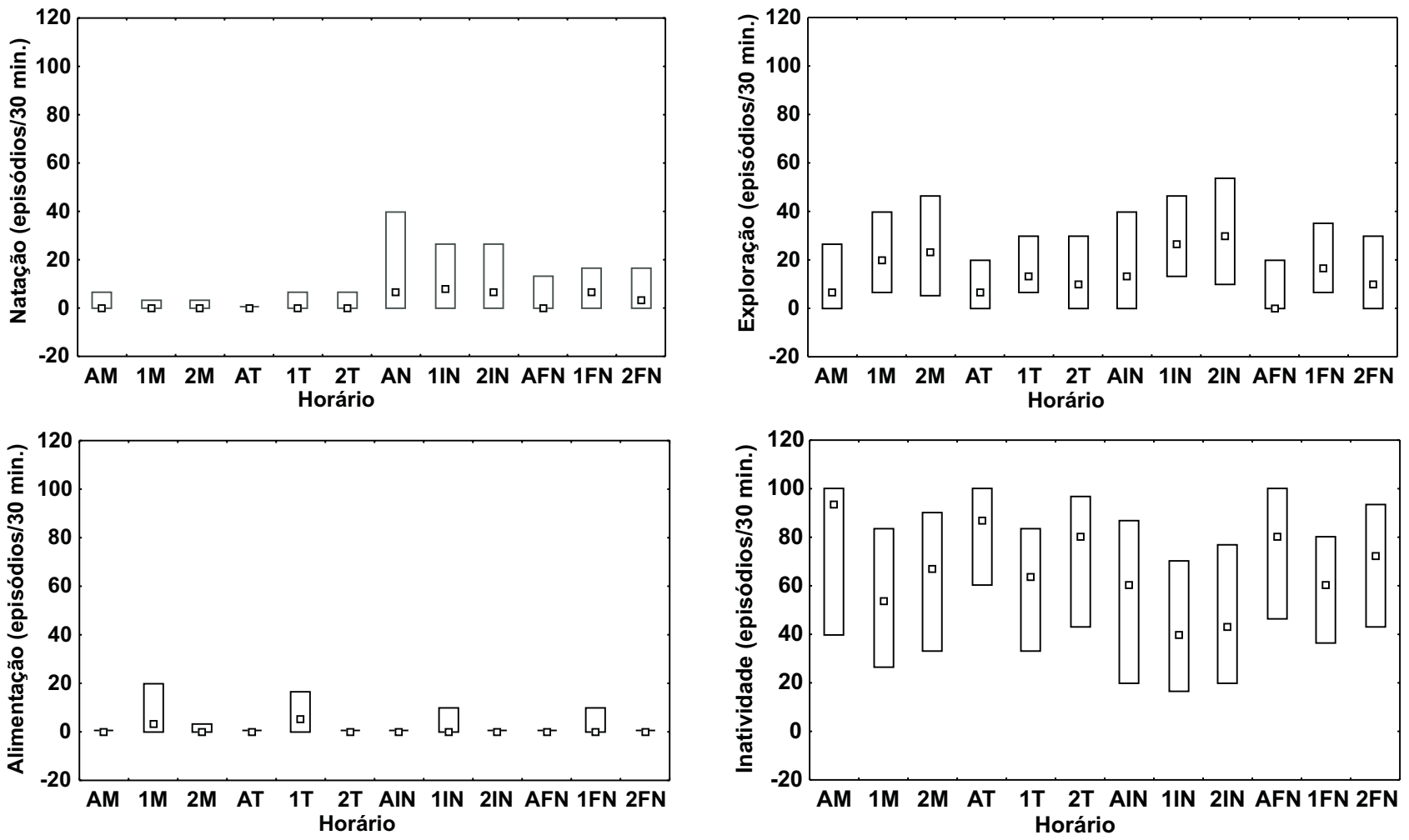

Figura 1. Comportamentos de natação, exploração, alimentação e inatividade de L. vannamei em função da oferta do alimento, de acordo com o horário de observação: (A) antes da oferta do alimento, (1) imediatamente após, (2) uma hora após, (M) manhã, (T) tarde, (IN) início da noite, (FN) final da noite.

A freqüência de inatividade dos animais sofreu modificações em função da presença do alimento artificial (Kruskal-Wallis, $\mathrm{H}(9,45)=382,6 ; \mathrm{p}=0,00)$. Os indivíduos mostraram-se mais inativos em todos as observações ocorridas antes (A) da oferta, quando confrontadas com a primeira (1) e segunda (2) meia hora após. Além disso, as primeiras meias horas (1) resultaram em menores percentuais que as segundas (2) (U de Mann-Whitney) (Fig. 1).

\section{DISCUSSÃO}

Observamos que, de uma maneira geral, as atividades comportamentais de L. vannamei sofrem influência presença do alimento artificial, variando em função da fase do dia, do horário de oferta e do tempo de exposição da ração. A natação ocorreu principalmente na fase escura e caracterizou-se como o único comportamento avaliado a não sofrer interferência significativa da presença do alimento. A exploração do substrato foi intensificada após a oferta da ração, em todos os horários das fases clara e escura, indicando uma relação direta com a busca do alimento. A alimentação mostrou-se mais freqüente na meia hora imediatamente posterior à oferta e ocorreu mais intensamente nos horários da fase clara com relação à escura.

Diversas pesquisas exploram a descrição hierárquica es- pecífica do comportamento alimentar de peneídeos, desencadeado a partir da oferta do alimento artificial acrescido de atrativos químicos de baixo peso molecular, geralmente aminoácidos livres (Harpaz \& Steiner 1990, Costero \& Meyers 1993, Pittet et al. 1996, Lee \& Meyers 1996). No entanto, não existem investigações, situadas no campo da etologia aplicada, sobre variações relacionadas ao repertório comportamental dos peneídeos expostos ao alimento artificial, de forma que comparações só poderão ser realizadas em função de parâmetros indiretamente relacionados.

Corroborando nossos resultados sobre a alimentação, por nós caracterizada como ingestão do alimento, NuNEs et al. (1996) observaram que o camarão marinho Farfantepenaeus subtilis (Pérez-Farfante, 1967) se alimenta tanto durante o dia quanto à noite, havendo sempre o incremento da ingestão, tanto do alimento artificial quanto do natural, após a ração ter sido distribuída no viveiro. Estes dados apontam que, no ambiente de cultivo, a oferta da ração induz à procura e ingestão de itens alimentares, o que vem reforçar nossos resultados relativos à exploração do substrato. Além disto, para F. subtilis, a alimentação é mais intensa nos 10 minutos posteriores à exposição à ração, ocorrendo após esta fase de forma interrupta e pausada (Nunes et al. 1997), verificando-se uma maior ocorrência de ali- 
mento no trato digestivo trinta minutos após a distribuição (Nunes \& Parsons 1999).

Nossa pesquisa indicou uma maior freqüência de alimentação na fase clara, na meia hora imediatamente posterior à oferta. Da mesma forma, SicK et al. (1973), constataram que em juvenis de Litopenaeus setiferus (Linnaeus, 1767) a taxa de ingestão de ração é diretamente proporcional à intensidade luminosa e ainda inversamente proporcional ao tempo de exposição ao alimento.

Pesquisas sobre atividade de camarões realizadas em laboratório costumam utilizar medição automática desta variável, quantificada através da movimentação como um todo (Hindley 1975, Guerao \& Ribera 1996). Informações específicas sobre peneídeos afirmam que a maioria possui sulco adrostral e apresenta padrão similar de atividade (ROBERTSON et al. 1993), sendo tipicamente ativos à noite e se enterrando durante o dia (HindLey 1975). Em contraste, espécies de camarões brancos que não possuem sulco adrostral, incluindo L. vannamei, são ativas tanto durante o dia quanto à noite, e não costumam se enterrar (Pérez-Farfante 1969). Não existem estudos de caracterização do repertório comportamental que compõe o padrão de atividade destes animais ao longo de $24 \mathrm{~h}$.

Com relação aos comportamentos exibidos por L. vannamei na presença do alimento artificial, vimos que se tornam mais ativos durante a primeira meia hora subseqüente à oferta, o que evidencia o incremento das atividades estudadas, em maior ou menor grau, em todos os horários do claro e escuro. Pela manhã, à tarde e final da noite, os animais, que antes da oferta encontravam-se preferencialmente inativos, passam a explorar e se alimentar. Somado a isto, a natação predomina na fase escura e a ingestão do alimento é mais acentuada na fase clara do dia.

É necessário ponderar que a oferta da ração na fase escura onera os gastos com manejo alimentar e também ocasiona a diminuição nos níveis de oxigênio dissolvido, em função da oxidação da matéria orgânica gerada pela ração adicionada ao viveiro. Considerando os fatores ambientais, econômicos e comportamentais envolvidos, recomendamos que o alimento artificial seja ministrado na fase clara do dia.

\section{AGRADECIMENTOS}

À Capes, Banco do Nordeste do Brasil, Cooperativa de Criadores Camarão do Rio grande do Norte, Fazenda de Carcinicultura Papeba e ao Departamento de Fisiologia da UFRN.

\section{REFERÊNCIAS BIBLIOGRÁFICAS}

Costero, M.T. \& S. Meyers. 1993. Evaluation of chemoreception by Penaeus vannamei under experimental condition. Progressive Fish-Culturist, Washington, 55 (3): 157-162.

Cuzon, G.; A. Lawrence; G. Gaxiola; C. Rosas \& J. Guillaume. 2004. Nutrition of Litopenaeus vannamei reared in tanks or in ponds. Aquaculture, Amsterdam, 235: 513-551.

Guerao, G. \& C. Ribera. 1996. Locomotor activity patterns and feeding habits in the prawn Palaemon serratus (Pennant, 1777) (Decapoda, Palaemonidae) in the Alfa Bay, Elbro Delta, Spain. Crustaceana, Leiden, 69 (1): 101-112.

Harpaz, S. \& J.E. STEINER. 1990. Analysis of betaine induced feeding behavior on the prawn Macrobrachium rosenbergii (DeMan 1879) (Decapoda, Caridea). Crustaceana, Leiden, 58: 175-185.

Hindley, J.P.R. 1975. Effects of Endogenous and Some Exogenous Factors on the Activity of Juvenile banana Prawn Penaeus merguiensis. Marine Biology, Berlin, 29: 01-08.

HuntingFord, F. 1991. The Study of Animal Behaviour. Padstow, T.J. Press, $3^{\text {rd }}$ ed., 367p.

Lee, P.G. \& S.P. Meyers. 1996. Chemoattraction and feeding stimulation in crustaceans. Aquaculture Nutrition, Oxford, 2: 157-164.

Martinez-Cordova, L.R.; A. Porchas-Cornejo; H. VillarrealColemnares; J.A. Calderon-Perez \& J.N. Naranjo-Paramo. 1998. Evaluation of Three Feeding Strategies on the Culture of White Shrimp Penaeus vannamei Boone 1931 in low water exchange ponds. Aquacultural Engineering, Amsterdam, 17: 21-28.

Nunes, A.J.P. \& G.J. Parsons. 1999. Feeding Levels of the Southern Brown Shrimp Penaeus subtilis in Response to Food Dispersal. Journal of the World Aquaculture Society, Baton Rouge, 30 (3): 331-348.

Pérez-Farfante, I. 1969. Western Atlantic shrimp of the genus Penaeus. Fishery Bulletin, Seattle, 67: 461-591.

Nunes, A.J.P.; S. Goddard \& T.C.V. Gesteira. 1996. Feeding activity patterns of the Southern brown shrimp Penaeus subtilis under semi-intensive culture in NE Brazil. Aquaculture, Amsterdam, 44: 371-386.

Nunes, A.J.P.; T.C.V. Gesteira \& S. Goddard. 1997. Food Ingestion and assimilation by the Southern Brown shrimp Penaeus subtilis under semi-intensive culture in NE Brazil. Aquaculture, Amsterdam, 149: 121-136.

Pittet, A.O., J.C. Ellis \& P.G. Lee. 1996. Methodology for the identification and quantitative measurement of chemical stimulants for penaeid shrimp. Aquaculture Nutrition, Oxford, 2: 75-182.

RoberTson, L., A.L. LAWRence \& F.L. CASTILle. 1993. Effect of feeding frequency and feeding time on Growth of Penaeus vannamei (Boone). Aquaculture and Fisheries Management, Reino Unido, 24: 01-06.

Rodriguez, G. \& E. NAYlor. 1972. Behavioural rhythms in littoral prawns. Journal of Marine Biology Association, Londres, 52: 81-95.

SEDGWICK, R.W. 1979. Effect of ration size and feeding frequency on the growth and food conversion of juvenile Penaeus merguiensis De Man. Aquaculture, Amsterdam, 16: 279-298.

SiCK, L.V.; D. White \& G. BAPTIST. 1973. The effect of duration of feeding, amount of food, light intensity, and animal size on rate of ingestion of pelleted food by juvenile penaeid shrimp. Progressive Fish-Culturist, Washington, 35 (01): 22-26.

SMith, D.M.; M.A. Burford; S.J. TabretT; S.J. Irvin \& L. Ward. 2002. The effect of feeding frequency on water quality and growth

Revista Brasileira de Zoologia 22 (3): 648-652, setembro 2005 
of the black tiger shrimp (Penaeus monodon). Aquaculture, Amsterdam, 207: 125-136.

Steel, R.G.D. \& J.H. Torrie. 1988. Bioestadística: principios e procedimientos. Mexico, McGraw-Hill, $2^{\mathrm{a}}$ ed., 622p.

Tacon, A.J.G.; J.J. Cody; L.D. Conquest; S. Divakaran; I.P. Forster \& O.E. DECAMP. 2002. Effect of culture system on the nutrition and growth performance of Pacific White shrimp Litopenaeus vannamei (Boone) fed different diets. Aquaculture Nutrition, Oxford, 8: 121-137.

Velasco, M.; A.L. Lawrence \& F.L. Castille. 1999. Effect of variations in daily feeding frequency and ration size on growth of shrimp Litopenaeus vannamei (Boone), in zerowater exchange culture tanks. Aquaculture, Amsterdam, 179: $141-148$.

Recebido em 25.X.2004; aceito em 05.VIII.2005. 